\title{
Thermo-mechanical responses of cracked quasi-transparent film to laser irradiation
}

\author{
Qing Peng ${ }^{\mathrm{a}}$, Chen-Wu Wu ${ }^{\mathrm{a}, *}$, Chen-Guang Huang ${ }^{\mathrm{a}, \mathrm{b}}$ \\ a Institute of Mechanics, Chinese Academy of Sciences, Beijing 100190, China \\ ${ }^{\mathrm{b}}$ School of Engineering Science, University of Chinese Academy of Sciences, Beijing 100049, China
}

\section{A R T I C L E I N F O}

\section{Keywords:}

Crack

Film

Thermal

Mechanical

Laser irradiation

\begin{abstract}
A B S T R A C T
Thermal and mechanical responses of a cracked transparent conducting film to laser irradiation were investigated. A representative coin-shaped crack was modeled in the transparent conducting film and the case of vertical incidence of laser beam considered. Firstly, the multiple reflections, transmissions as well as absorptions are formulated in the quasi-multilayer media due to the effect of inner crack. Then, the temperature characteristics generated by the dissipated light energy are computed for the film of typical thermal boundary conditions. Finally, the thermal stress and stress intensity factors around the crack tip are particularly analyzed. The effects of the coin-shaped crack are discussed on the multi-physical responses of the film to laser irradiation.
\end{abstract}

\section{Introduction}

The transparent conducting film of Al-doped $\mathrm{ZnO}$ (AZO film) have been largely used as in the photovoltaic cells, waveguides and many other important photoelectric devices because of its excellent performance and relatively low price [3,13,10,33,6,23,7,31,9]. Of course, the AZO film is actually not absolute transparent as there is necessarily absorption of light transmitting through it. The electrical conductivity as well as surface morphology of Al-doped $\mathrm{ZnO}$ film could be enhanced obviously by increasing its thickness [1]. Although the rough surface of AZO layer could lead to inner discontinuity, which could act as defects especially when the bi-layer structure of AZO/ZnO is taken into concept [17]. These defects are always strictly unacceptable in optical materials as the discontinuity would increase the reflection and scattering of the incident light and dissipate much light energy into heat, which means that unexpected temperature elevation and thermal stress would arise in the structure. Such thermo-mechanical responses would slightly change with the absorption of guided waves if the wave-guide structures are taken into account. Therefore, the correct mathematical descriptions are required on the characteristics of temperature, thermal stress as well as its possible singularity to estimate the reliability of a device involving such AZO film, in particular when tiny flaws appear.

The thermo-mechanical properties of the intact AZO film has been modeled based on the public data distributed in the large literatures [18,8,11 and 21]. However, subsurface or interface discontinuity could be developed in the optical glasses as well as various surface functional films by many processing wherein the contact pressure is involved, or as aforementioned [5,20,22,34]. At the same time, the responses, such as scattering and intensification of the flaws to the incident light have also been utilized to detect them $[27,40]$. In these detection methods, the mechanical stress effect has also attracting much attention [28]. Although, the full physical mechanism on the interaction between a subsurface crack and light irradiation has not been revealed thoroughly except for some simplified model that approximating the incident light energy as simple thermal loadings without considering the reflection,

\footnotetext{
* Corresponding author at: Institute of Mechanics, Chinese Academy of Sciences, No.15 BeisihuanXi Road, Beijing 100190, China.

E-mail addresses: chenwuwu@imech.ac.cn, c.w.wu@outlook.com (C.-W. Wu).
} 


\section{Nomenclature}

$A_{0} \quad$ amplitude of the plane harmonic wave

$A_{k} \quad$ amplitude of the reflected beams

$A_{k}^{t} \quad$ amplitude of the transmitted beams

a model coefficients

$b \quad$ model coefficients

$C_{i} \quad$ model coefficients

c specific capacity

$D \quad$ thermal diffusivity

$D_{i} \quad$ computing domains

$d \quad$ thickness of the sample

$d_{1} \quad$ crack location, distance from the irradiated surface to the crack location

$d_{2} \quad$ distance from the bottom surface to the crack

$d_{m} \quad$ center value of $d_{1}$ for the averaged calculations

E elastic modulus

$E_{i} \quad$ energy flow

$E_{I} \quad$ effective energy density of the incident laser

$h$ convective heat transfer coefficient, $10 \mathrm{~W} / \mathrm{m}^{2} / \mathrm{K}$

$K_{c} \quad$ characteristic Stress intensity factor

$K_{I} \quad$ stress intensity factor of type I

$\left[K_{I}\right]$ dimensionless Stress intensity factor of type I

$K_{I I} \quad$ stress intensity factor of type II

$\left[K_{I I}\right]$ dimensionless Stress intensity factor of type II

$k_{c} \quad$ thermal conductivity

$n \quad$ outward normal unit vector of the surface

$n_{i} \quad$ complex refraction index of the medium $i$

$Q \quad$ heat generation rate

$Q_{c} \quad$ characteristic heat generation rate

$Q_{i} \quad$ heat generation rate per unit volume for domain

$\left[Q_{i}\right]$ dimensionless heat generation rate per unit volume for domain

$R \quad$ radius of the sample

$R_{c} \quad$ radius of the coin-shape crack

$R_{b} \quad$ radius of the laser beam $r_{f}$

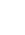

$t$

$T$

$T_{c}$

$\left[T_{\max }\right]$ dimensionless maximum temperature

$T_{\max } \quad$ maximum temperature

$T_{a m b} \quad$ ambient temperature, $20^{\circ} \mathrm{C}$

$\boldsymbol{u}$ displacement vector

$\alpha \quad$ thermal expansion coefficient

$\beta_{i} \quad$ model coefficients

$\theta_{i} \quad$ angle in medium $i$

$\lambda$ laser wavelength

$\rho$ density

$v \quad$ Poisson's ratio $\epsilon$

$\sigma$

$\sigma_{x y}$

$\sigma_{y y}$

$\sigma_{c}$

$\left[\sigma_{i j}\right]$

surface emissivity, 0.85

Stefan-Boltzmann constant

$x y$-component of the stress tensor

$y y$-component of the stress tensor

characteristic stress

Definitions of the dimensionless parameters

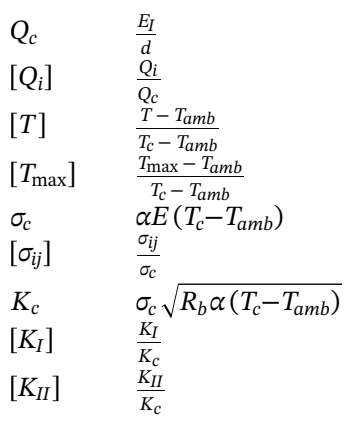

transmission of the light at the cracks [37].

Laser induced damage in such quasi-transparent optical materials, or commonly known as glass materials as well as the interaction between laser and the flaws in these materials play significant role in optical engineering, especially when the multidisciplinary design optimization is considered wherein light, thermal and mechanical reliability are targeted simultaneously $[24,25,32,35]$.

Actually, the interaction between monochromatic light and opaque material is largely discussed in the field of laser manufacturing [19,12], wherein the temperature, phase change and stress are particularly focused on [29,26]. For materials with a

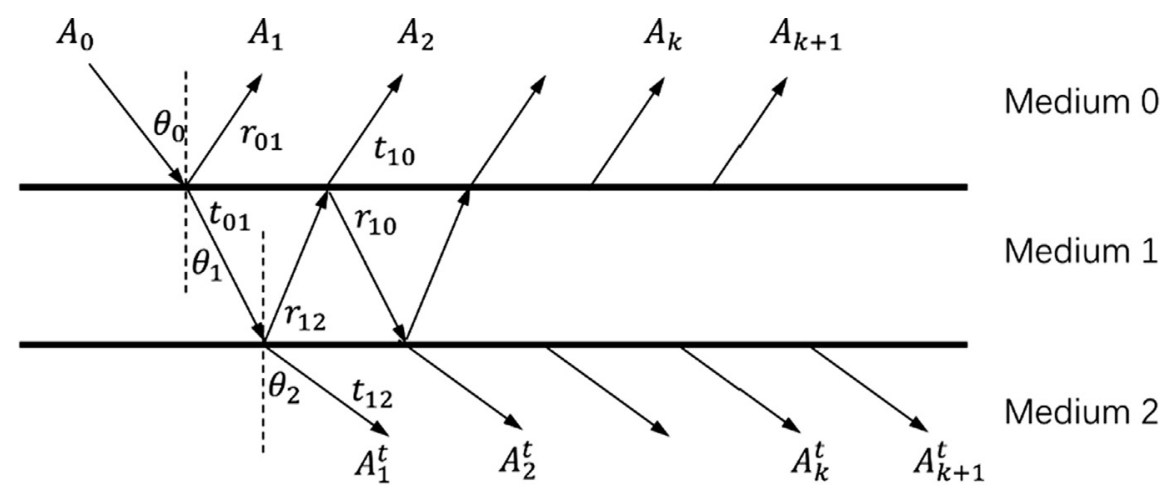

Fig. 1. Sketch of monochromatic light propagation through homogeneous medium. 
relatively low extinction coefficient, such as glass and quasi-transparent metal oxide, the penetration depth of laser beam in such materials is much larger than opaque one. Modeling laser irradiation as a surface flux would deviate the mathematical model from the actual physical process and detailed description on the volumetric heat generation is required. Actually, the volumetric heat generation resulted from laser irradiation has been investigated extensively in the intact medium $[4,2,38,36]$.

However, in the presence of defects such as a crack in the medium [15,16,39], energy absorption would be obviously altered because of the reflections at the interface of the medium and the defects. The effect of the defects on volumetric heat source should be carefully inspected to obtain an accurate solution.

In the present work, a single layer of AZO with a crack in the center subjected to a vertically incident laser beam irradiation is investigated. Reflections and transmissions of the laser, as well as interferences of the laser beams, are taken into account. The volumetric heat generation rate due to laser irradiation is formulated, based on which a thermal field is calculated with finite element method. The effects of such thermal field on the induced stress field in the presence of the crack are discussed.

\section{Theoretical formula on volumetric heat generation rate}

Fig. 1 illustrates the modeling concept developed by Wu et al. [36,38] on the propagation of the monochromatic light in a multilayer media. Many derivations that included in the above reference are rearranged herein to improve the readability of the present work.

A plane simple harmonic electromagnetic wave, with an amplitude $A_{0}$, impinges at the planar interface separating the incident medium (M0) and a second medium (M1) of finite thickness $d$. Without loss of generality, the third medium (M2) underneath the second medium is assumed generic. The complex refraction index for each medium $\mathrm{Mi}, i=0,1,2$ is $n_{i}-j k_{i}$ and the incident angle in M0 is $\theta_{0}$. Herein, we need establish the overall description on the reflection, absorption and transmission of the incident monochromatic light through the medium M2.

The vector summation over all of the spatially overlapped rays is required to calculate the amplitude of the total reflection and the transmission waves. The amplitudes of the reflection rays and the transmission rays are denoted by $A_{i}$ and $A_{i}^{t}$ respectively. The summation of amplitudes for all reflection rays and all transmission rays could be expressed as follows, respectively [38,36].

$$
\begin{aligned}
& \frac{A_{R}}{A_{0}}=\frac{1}{A_{0}} \sum_{k=1}^{\infty} A_{k}=r_{01}+t_{01} r_{12} t_{10} e^{-j 2 \delta_{1}} e^{-2 \xi_{1}}+\cdots+t_{01}\left(r_{12} r_{10}\right)^{k-2} r_{12} t_{10} e^{-j 2(k-1) \delta_{1}} e^{-2(k-1) \xi_{1}}+\cdots=\frac{r_{01}+r_{12} e^{-j 2 \delta_{1}} e^{-2 \xi_{1}}}{1+r_{01} r_{12} e^{-j 2 \delta_{1}} e^{-2 \xi_{1}}} \\
& \frac{A_{T}}{A_{0}}=\frac{1}{A_{0}} \sum_{k=1}^{\infty} A_{k}^{t}=t_{01} t_{12} e^{-j \delta_{1}} e^{-\xi_{1}}+\cdots+t_{01}\left(r_{12} r_{10}\right)^{k-1} t_{12} e^{-j(2 k-1) \delta_{1}} e^{-(2 k-1) \xi_{1}}+\cdots=\frac{t_{01} t_{12} e^{-j \delta_{1}} e^{-\xi_{1}}}{1+r_{01} r_{12} e^{-j 2 \delta_{1}} e^{-2 \xi_{1}}}
\end{aligned}
$$

Where,

$$
\begin{aligned}
& \delta_{1}=\frac{2 \pi}{\lambda} n_{1} d_{1} \cos \theta_{1} \\
& \xi_{1}=\frac{2 \pi k_{1}}{\lambda} n_{1} d_{1}
\end{aligned}
$$

With $r_{01}$ being the reflected ratio in amplitudes at interface M0-M1 from M0 side and $r_{10}$ M1 side, $t_{01}$ the transmitted ratio across interface from M0 to M1 and $t_{10}$ from M1 to M0.

By introducing the optical admittance $\eta$, the reflected and transmitted ratios can be written as

$$
\left\{\begin{array}{l}
r_{i j}=\frac{\eta_{i}-\eta_{j}}{\eta_{i}+\eta_{j}} \\
t_{i j}=\frac{2 \eta_{i}}{\eta_{i}+\eta_{j}} \zeta_{i j}
\end{array}\right.
$$

where,

$$
\eta=\left\{\begin{array}{cl}
\frac{N}{\cos \theta}, & \text { for } \mathrm{P} \text { - wave } \\
N \cos \theta, & \text { for S-wave }
\end{array}\right.
$$

and

$$
\zeta_{i j}=\left\{\begin{array}{cc}
\frac{\cos \theta_{i}}{\cos \theta_{j}}, & \text { for P- wave } \\
1, & \text { for S-wave }
\end{array}\right.
$$

Then after, the energy flux for reflection and transmission could be formulated. Denoting $R$ and $T$ as the reflection ratio and the transmission ratio on energy flow, one can finally get $[38,36]$

$$
R=\left|\frac{A_{R}}{A_{0}}\right|^{2}=\frac{r_{01}^{2}+r_{12}^{2} e^{-4 \xi_{1}}+2 r_{01} r_{12} e^{-2 \xi_{1}} \cos 2 \delta_{1}}{1+\left(r_{01} r_{12}\right)^{2} e^{-4 \xi_{1}}+2 r_{01} r_{12} e^{-2 \xi_{1}} \cos 2 \delta_{1}}
$$




$$
T=\left|\frac{A_{T}}{A_{0}}\right|^{2} \frac{n_{2} \cos \theta_{2}}{n_{0} \cos \theta_{0}}=\frac{\left(t_{01} t_{12}\right)^{2} e^{-2 \xi_{1}}}{1+\left(r_{01} r_{12}\right)^{2} e^{-4 \xi_{1}}+2 r_{01} r_{12} e^{-2 \xi_{1}} \cos 2 \delta_{1}} \frac{n_{2} \cos \theta_{2}}{n_{0} \cos \theta_{0}}
$$

Since both $r_{i j}$ and $t_{i j}$ differ for P-wave and S-wave, the energy flux ratios should be calculated separately for P-wave and S-wave. Then, the total energy flux ratios can be obtained by adding up the two parts with the following scalar operations.

$$
\begin{aligned}
& R=\frac{1}{2}\left(R_{P}+R_{S}\right) \\
& T=\frac{1}{2}\left(T_{P}+T_{S}\right)
\end{aligned}
$$

where the subscription $\mathrm{P}$ and S denote the components for P-wave and S-wave, respectively. Therefore, the heat generation rate $Q$ can be obtained through the equation of energy balance as following by assuming that all of the absorbed light energy dissipates into heat immediately.

$$
Q V=E_{I}(1-R-T)
$$

where in $V$ represents the volume of the heated region and $E_{I}$ the energy flow of the incident monochromatic light.

Fig. 2 illustrates the model on the cracked AZO film for the case when a planar crack exists in the medium, dividing the medium into layer 1 and layer 2. Herein, medium 0 and medium 3 refer to the air environment. We can assume the space enclosed by the crack surfaces as another virtual layer of medium denoted by medium c, in particular when the opening displacement of the coinshape crack is very small. This is the very case considered in the present work. Accurately enough, the thickness of the medium $\mathrm{c}$ is assumed to be zero and, let $R_{c 2 c}$ represent the reflection originated in medium c on the interface c-2, and then back into medium c, $T_{c 23}$ the transmission from medium c, through medium 2 (layer 2), and into medium 3 (substrate). It is conceivable that the absorption in the crack layer would be zero and optical interference does not occur in such a virtual layer.

For Layer 1 , the absorbed light energy rate $E_{1}$ can be expressed as

$$
\frac{E_{1}}{E_{0}}=\left(1-R_{010}-T_{01 c}\right)+\sum_{k=2}^{\infty} T_{01 c}\left(R_{c 2 c} R_{c 1 c}\right)^{k-2} R_{c 2 c}\left(1-R_{c 1 c}-T_{c 10}\right)
$$

Then the net heat generation per unit time per unit volume in layer 1 is

$$
Q_{1}=\frac{E_{1}}{V_{1}}=\left(\left(1-R_{010}-T_{01 c}\right)+\frac{T_{01 c} R_{c 2 c}\left(1-R_{c 1 c}-T_{c 10}\right)}{1-R_{c 2 c} R_{c 1 c}}\right) \frac{E_{0}}{V_{1}}
$$

where $V_{1}$ is the volume of layer 1 . Similarly, the absorbed light energy rate $E_{2}$ for layer 2 can be obtained as

$$
\frac{E_{2}}{E_{0}}=\sum_{k=1}^{\infty} T_{01 c}\left(R_{c 2 c} R_{c 1 c}\right)^{k-1}\left(1-R_{c 2 c}-T_{c 23}\right)
$$

The net heat generation per unit time per unit volume in layer 2 is

$$
Q_{2}=\frac{E_{2}}{V_{2}}=\frac{T_{01 c}\left(1-R_{c 2 c}-T_{c 23}\right)}{1-R_{c 2 c} R_{c 1 c}} \frac{E_{0}}{V_{2}}
$$

where $V_{2}$ is the thickness of layer 2 .

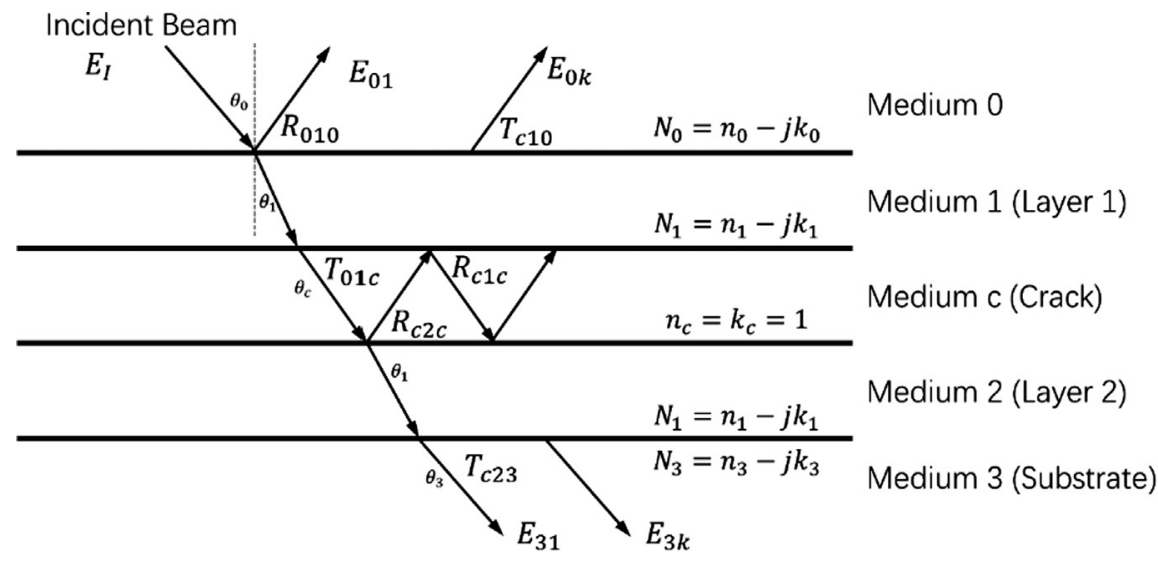

Fig. 2. Illustration of monochromatic light propagation through cracked medium. 


\section{FEM model on temperature and stress}

To calculate the temperature field and stress intensity factors of the coin-shape crack in an AZO film irradiated by a laser beam, an axisymmetric FEM model as shown in Fig. 3 is set up to solve the heat conduction equations as well as the thermo-elastic mechanics equations

$$
\frac{1}{D} \frac{\partial T}{\partial t}-\nabla^{2} T=\frac{Q}{k_{c}}
$$

And

$$
\frac{3(1-v)}{1+\nu} \nabla \nabla \cdot \boldsymbol{u}-\frac{3(1-2 \nu)}{2(1+v)} \nabla \times \nabla \times \boldsymbol{u}=\alpha \nabla T
$$

The thermal boundary conditions of

$$
\begin{aligned}
& k_{c} \frac{\partial T}{\partial \boldsymbol{n}}+h\left(T-T_{a m b}\right)=0 \\
& k_{c} \frac{\partial T}{\partial \boldsymbol{n}}+\epsilon \sigma\left(T^{4}-T_{a m b}^{4}\right)=0
\end{aligned}
$$

are applied to all of the outward surfaces while the adiabatic conditions are assumed on the crack surfaces.

A cylindrical coordinate system is adopted with the origin being coincident with the intersection point of the laser beam centerline and the irradiated surface of the film sample, and z-axis is the symmetry axis with symmetry boundary conditions.

Moreover, the displacement boundary conditions of

$$
u_{z}=0
$$

Was applied to the bottom surface of the model for a rigid substrate.

The laser beam with radius $R_{b}$ and output energy rate (power) is assumed to be perpendicular to the irradiated surface, which means that $\theta_{0}=0$ in all of the formulae in the previous chapter. The thickness and radius of the film sample is denoted with $d$ and $R$, respectively. A coin-shape crack assumed to be located at $z=d_{1}$ and of half-length $R_{c}$.

The overall computational domain can be divided into four adjacent subdomains as following

$$
\begin{aligned}
& D_{0}=\left\{r, z \mid R_{c} \leqslant r<R_{b},-d \leqslant z \leqslant 0\right\} \\
& D_{1}=\left\{r, z \mid r<R_{c},-d_{1} \leqslant z \leqslant 0\right\} \\
& D_{2}=\left\{r, z \mid r<R_{c},-d \leqslant z<-d_{1}\right\}
\end{aligned}
$$

and

$$
D_{3}=\left\{r, z \mid r \geqslant R_{b}\right\}
$$

Apparently, if the crack radius $R_{c}$ exceeds the laser beam radius $R_{b}$, domain $D_{0}$ vanishes. Table 1 lists the detailed parameters used for the simulation.

Variations on both crack location $d_{1}$ and crack radius $R_{c}$ and their effects on the thermos-mechanical behaviors of the sample are specifically analyzed. The effects of the various crack location would work at two different scales, in which the long-range effect account for light absorption path length and the short-range for the light interference pattern. To study both the long-range effect and the short-range effect of the crack location on, the crack location $d_{1}$ is set to be a function of light wavelength as follows:

$$
d_{1}=10 \beta_{1}+0.05 \beta_{2} \lambda
$$

where $\beta_{1}$ and $\beta_{2}$ are integers successively chosen from 2 to 8 and from -10 to 10 , respectively. The radius of the coin-shape crack radius is set as $50 \mu \mathrm{m}, 100 \mu \mathrm{m}, 150 \mu \mathrm{m}, 200 \mu \mathrm{m}, 250 \mu \mathrm{m}, 300 \mu \mathrm{m}, 400 \mu \mathrm{m}, 500 \mu \mathrm{m}, 600 \mu \mathrm{m}, 1000 \mu \mathrm{m}$ and $1500 \mu \mathrm{m}$ in succession. Therefore, total 1617 calculation cases are performed in the present work to reveal the latent parameter dependency of the thermo-

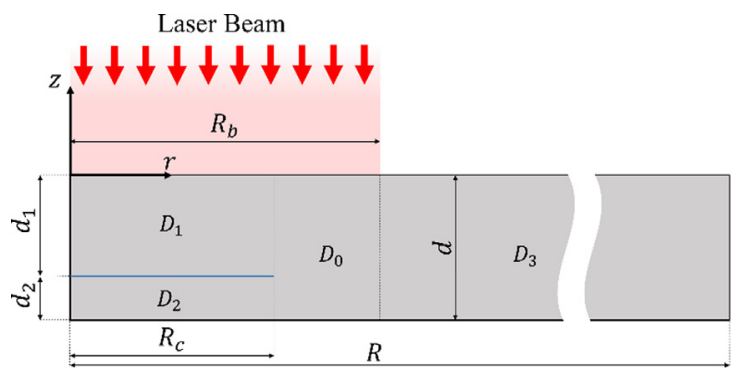

Fig. 3. Sketch of FEM model on thermal and mechanical responses. 
Table 1

Parameters used in the FEM model.

\begin{tabular}{ll}
\hline Parameter & Value \\
\hline Laser wavelength $\lambda$ & $523 \mathrm{~nm}$ \\
Effective power density $E_{I}$ & $1 \mathrm{e} 6 \mathrm{~W} / \mathrm{m}^{2}$ \\
Radius of laser beam $R_{b}$ & $250 \mu \mathrm{m}$ \\
Refraction index of environment (medium 0 ) $n_{0}$ & $1-0 j$ \\
Refraction index of sample $n_{1}$ & $1.8699-0.0014486 j$ \\
Refraction index of crack $n_{c}$ & $1-0 j$ \\
Refraction index of substrate $n_{S}$ & $1-0 j$ \\
Calculation domain $R \times d$ & $3000 \mu \mathrm{m} \times 100 \mu \mathrm{m}$ \\
Conductivity $k_{c}$ & $4.4 \mathrm{~W} / \mathrm{mK}$ \\
Specific capacity $c$ & $480 \mathrm{~J} / \mathrm{kgK}$ \\
Density $\rho$ & $5670 \mathrm{~kg} / \mathrm{m}^{3}$ \\
Thermal expansion $\alpha$ & $7.2 \times 10^{-6}$ \\
Elastic modulus $E$ & $110 \mathrm{GPa}$ \\
Poisson ratio $\nu$ & 0.3 \\
\hline
\end{tabular}

mechanical responses.

\section{Results and discussion}

\subsection{Temperature}

Fig. 4 plots the three volumetric heat generation rates: $Q_{0}, Q_{1}$, and $Q_{2}$, for subdomain $D_{0}, D_{1}$, and $D_{2}$ respectively.

Obviously, $Q_{0}$ is independent of $d_{1}$ as only the case of vertical irradiation is considered herein. Due to the interferences between the incident and reflect rays, the volumetric heat generation rates $Q_{1}$, and $Q_{2}$ would oscillate and the mid-value decrease with increasing of crack location $d_{1}$. Several magnified insets in Fig. 4 show exactly the oscillatory patterns of both $Q_{1}$ and $Q_{2}$ around each typical crack location within a range of $\pm \lambda$. The gradual reduction in mean value of the volumetric heat generation rates with increasing of $d_{1}$ should be partially resulted from the increasing of absorption distance in the layer above the crack. The two limiting cases that $d_{1}=0$ and $d_{1}=100 \mu \mathrm{m}$ are in corresponding to the physical reality that there is no crack, for which $Q_{1}$ and $Q_{2}$ should be identical to $Q_{0}$, as shown in Fig. 4.

The maximum temperature resides at the top layer of the crack on the axisymmetric axis, as shown in the contour graph in Fig. 5 .

Fig. 6 shows the maximum temperature in the computational domain versus various crack locations and crack radii, wherein $d_{m}=20 \mu \mathrm{m}$ is the center value of investigated crack locations as a general example. For any given crack location and crack radius, the maximum temperature oscillates in a manner similar to that of volumetric heat generation rate. In the neighborhood of a crack location $d_{1}=d_{m}$, the oscillatory behavior of the maximum temperature can be approximated by the following sinusoidal function:

$$
T_{\max }=C_{0}+C_{1} \sin (a x+b)
$$

where $C_{0}$ represents the mid-value, $C_{1}$ the oscillation amplitude, $a$ the frequency and $b$ the starting phase.

The legend in Fig. 6 lists the fitted function for various crack radii, in which the oscillation wavelength is about $\lambda_{0} / 4$, much less than the total depth $(d)$ of the sample.

Fig. 7 shows the mid-value of maximum temperature, $C_{0}$ as a function of $R_{c} / R_{b}$, with error bars $\left(C_{1}\right)$ indicating the oscillation amplitude of $T_{\max }$. It is indicated that $C_{0}$ and $C_{1}$ increase quickly with $R_{c} / R_{b}$ when $R_{c} / R_{b} \leqslant 1$ and then apparently slowly when $R_{c} / R_{b}>1$, which means that the edge influence would be reduced if the crack tip is far away from the laser beam spot.

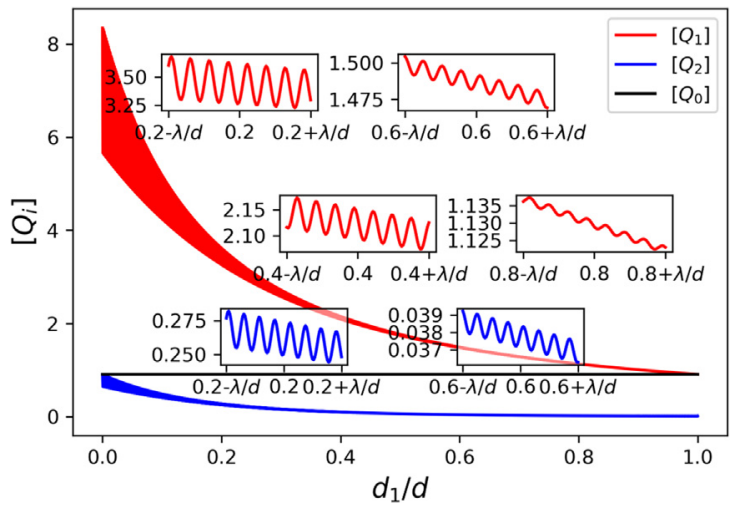

Fig. 4. Fluctuations of dimensionless volumetric heat generation rates $\left(\left[Q_{0}\right],\left[Q_{1}\right],\left[Q_{2}\right]\right)$ versus $d_{1} / d$. 


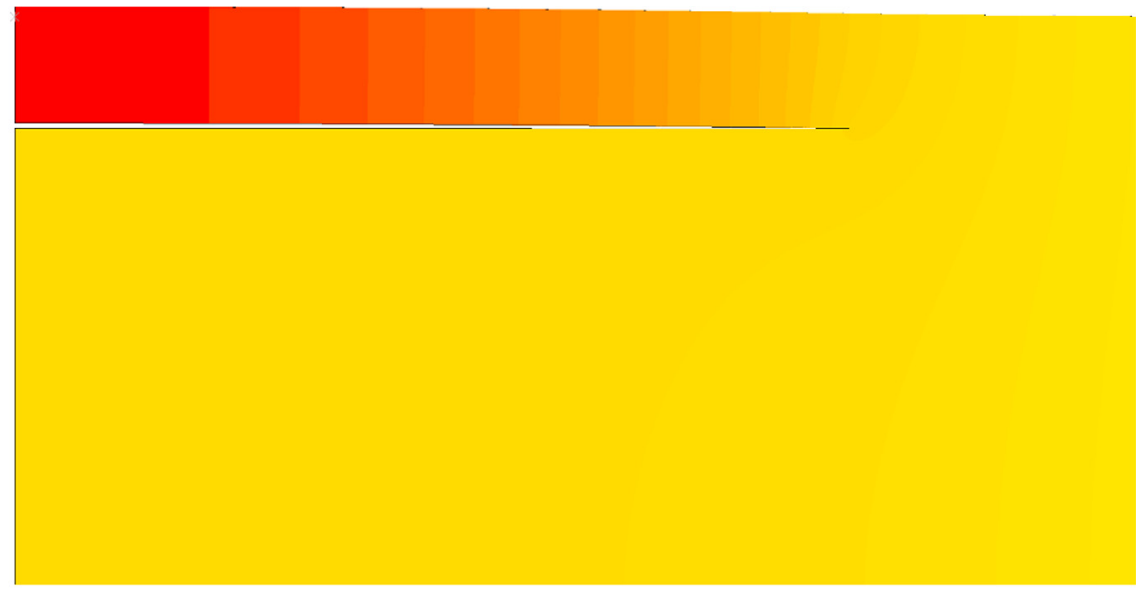

$\begin{array}{llllllllll}20 & 55.203 & 90.406 & 125.609 & 160.812 & 196.015 & 231.218 & 266.421 & 301.624 & 341.856\end{array}$

Fig. 5. Temperature field distribution near the crack $\left(d_{1}=20 \mu \mathrm{m}, R_{c}=150 \mu \mathrm{m}\right)$.

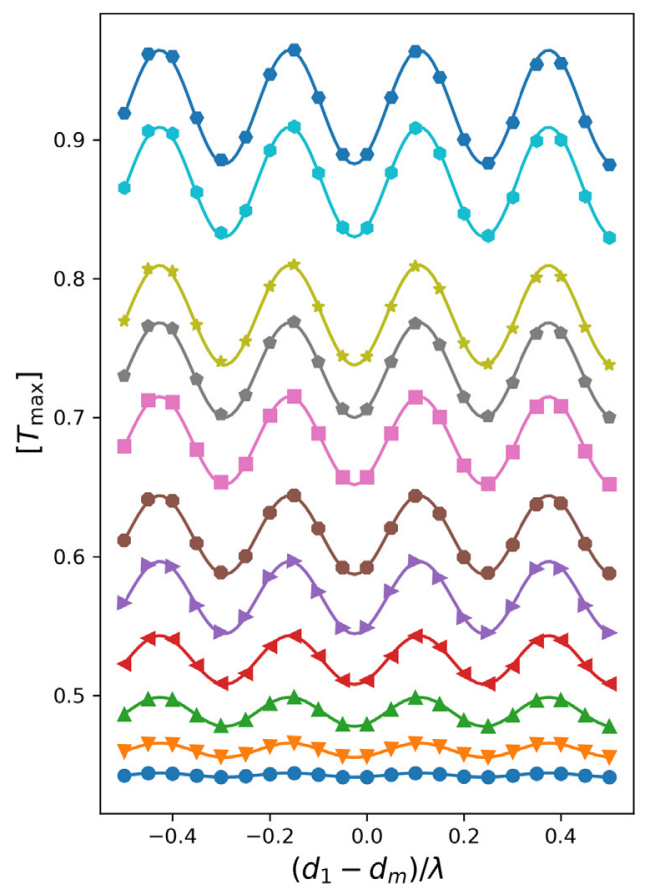

$R_{c}=50.0 \mu \mathrm{m}$

$y=0.443+0.001 \sin (23.510 x-0.976)$

$R_{\mathrm{c}}=100.0 \mu \mathrm{m}$

$\nabla \quad y=0.461+0.005 \sin (23.509 x-0.976)$

$\Delta R_{c}=150.0 \mu \mathrm{m}$

$\Delta y=0.488+0.010 \sin (23.509 x-0.975)$

$R_{c}=200.0 \mu \mathrm{m}$

$R_{c}=200.00 m$
$y=0.526+0.018 \sin (23.509 x-0.975)$

$R_{C}=250.0 \mu \mathrm{m}$

$R_{C}=0.570+0.026 \sin (23.508 x-0.975)$
$-R_{c}=300.00 m$

$R_{c}=300.0 \mu \mathrm{m}$

- $y=0.615+0.028 \sin (23.509 x-0.973)$

$R_{c}=400.0 \mu \mathrm{m}$

- $\begin{aligned} & R_{c}=400.0 \mu \mathrm{m} \\ & y=0.683+0.032 \sin (23.510 x-0.971)\end{aligned}$

$R_{c}=500.0 \mu \mathrm{m}$

- $y=0.734+0.034 \sin (23.510 x-0.970)$

* $R_{c}=600.0 \mu \mathrm{m}$

\# $y=0.774+0.036 \sin (23.511 x-0.970)$

$R_{c}=1000.0 \mu \mathrm{m}$

- $\begin{aligned} & R_{c}=1000.0 \mu m \\ & y=0.869+0.040 \sin (23.511 x-0.969)\end{aligned}$

$R_{c}=1500.0 \mu m$

$y=0.923+0.041 \sin (23.511 x-0.969)$

Fig. 6. Dimensionless maximum temperature as a function of $d_{1}$ in the neighborhood of $d_{m}=20 \mu m$.

Fig. 8 shows the dependency of the mid-value of maximum temperature $T_{\max }$ on the crack location $d_{1}$ for various crack radii. The error bar indicates the rapid oscillation of $T_{\max }$ as $d_{1}$ changes slightly around each typical point. For any specific crack radius, $T_{\max }$ would oscillate rapidly and the mid-value of $T_{\max }$ decreases with increase of $d_{1}$. As demonstrated by the data curves in Fig. 4, a larger $d_{1}$ means smaller values of $Q_{1}$ and $Q_{2}$, i.e. smaller volumetric heat generation rates. Thus for any given crack radius $R_{c}$, the maximum temperature $T_{\max }$ will decrease as $d_{1}$ increases. At the same time, for any given crack location $d_{1}$, a larger crack radius $R_{c}$ results in a higher maximum temperature. This is because the multiple reflections arise in a larger domain, which indicates more light energy would be absorbed and dissipated into heat.

\subsection{Stress}

Figs. 9 and 10 show the computed stress contours for the case that $d=20 \mu m$ and $R_{c}=150 \mu m$. 


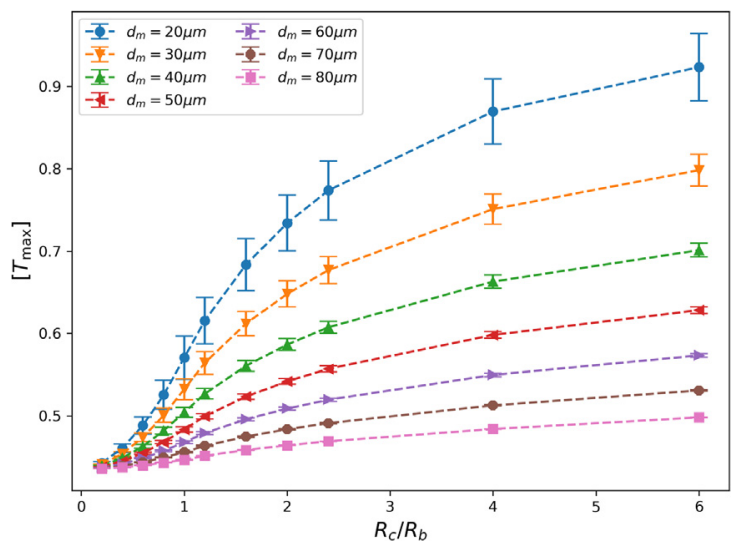

Fig. 7. Dimensionless maximum temperature versus $R_{c} / R_{b}$ for various crack location.

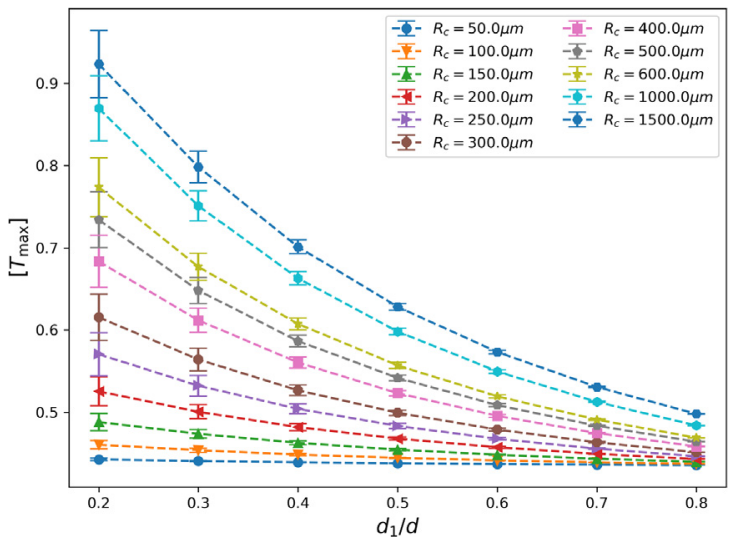

Fig. 8. Dimensionless Maximum temperature versus $d_{1} / d$ for various crack radius.

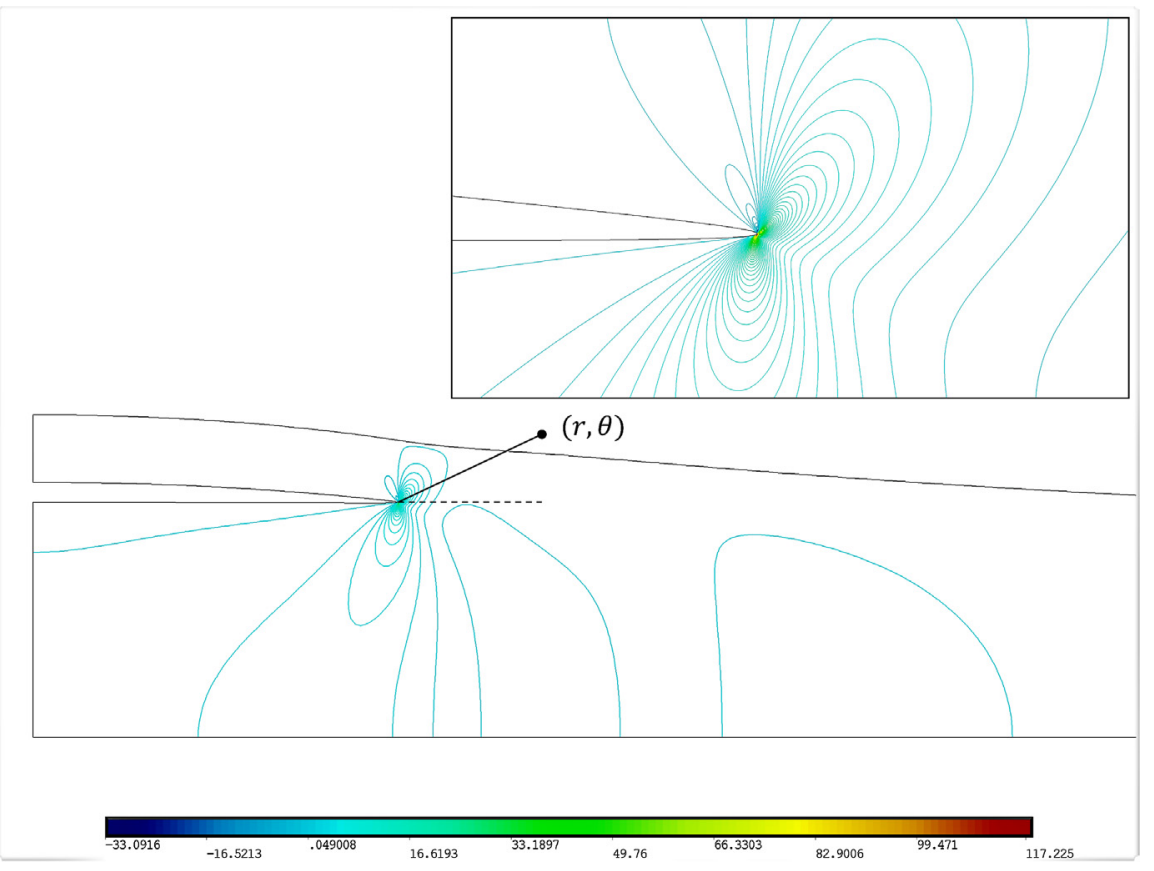

Fig. 9. Contour of $\sigma_{y y}$ for the case $\left(d_{1}=20 \mu m, R_{c}=150 \mu m\right)$. 


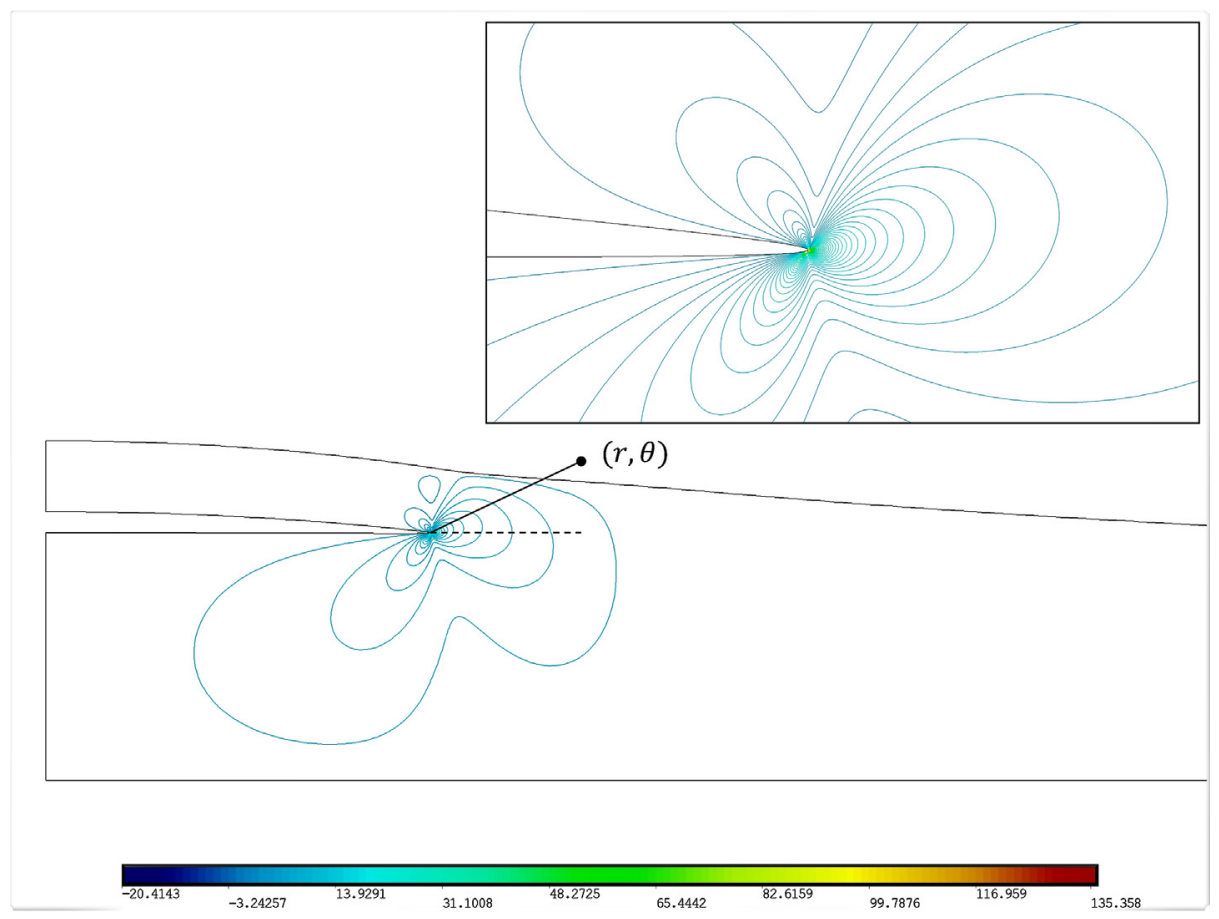

Fig. 10. Contour of $\sigma_{x y}$ for the case $\left(d_{1}=20 \mu m, R_{c}=150 \mu m\right)$.

A polar coordinate system is established with the origin located at the crack tip and the horizontal dash line coincident with $\theta=0$. It is shown that both stress components $\sigma_{y y}$ and $\sigma_{x y}$ are highly concentrated in a very small area of the crack tip, which is generally thought to be stress singularity behavior. Mathematically, the abrupt change of the stress components $\sigma_{y y}$ and $\sigma_{x y}$ versus the distance from the crack tip are determined by the stress intensity factors as following according to classic fracture mechanics.

$$
\begin{aligned}
& \sigma_{y y}=\frac{K_{I}}{\sqrt{2 \pi r}} \\
& \sigma_{x y}=\frac{K_{I I}}{\sqrt{2 \pi r}}
\end{aligned}
$$

where $K_{I}$ and $K_{I I}$ are mode I and mode II stress intensity factors, respectively. Therefore, the stress intensity factors $K_{I}$ and $K_{I I}$ could be calculated by fitting the stress data along any path pointing to the crack tip, for which the horizontal path mapping of the stress components for the present computation case are further shown in Fig. 11 as an example. Based on such numerical methods, the stress intensity factors $K_{I}$ and $K_{I I}$ are calculated for the typical cases and the parameter dependency extensively studied as the stress intensity factors dominate the defects behaviors.

Figs. 12 and 13 show the effects of the crack location $d_{1}$ on the stress intensity factors $K_{I}$ and $K_{I I}$, respectively.

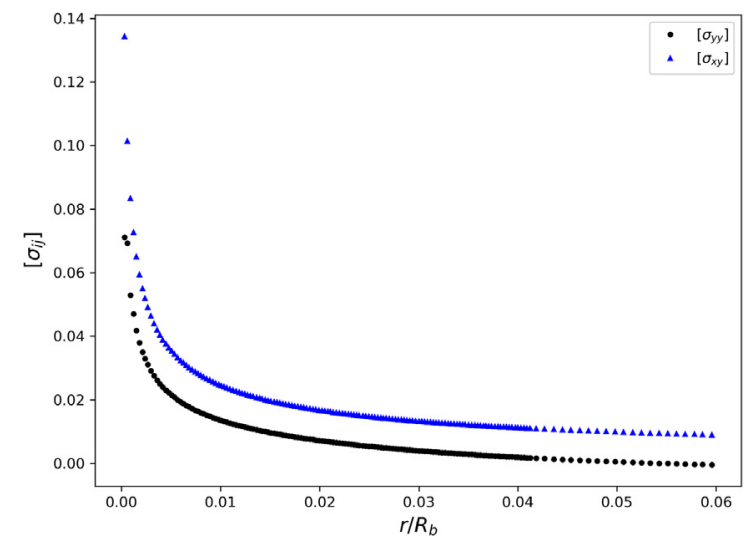

Fig. 11. Dimensionless stresses versus $r$ when $\theta=0$ for the case $\left(d=20 \mu m, R_{c}=150 \mu \mathrm{m}\right)$. 


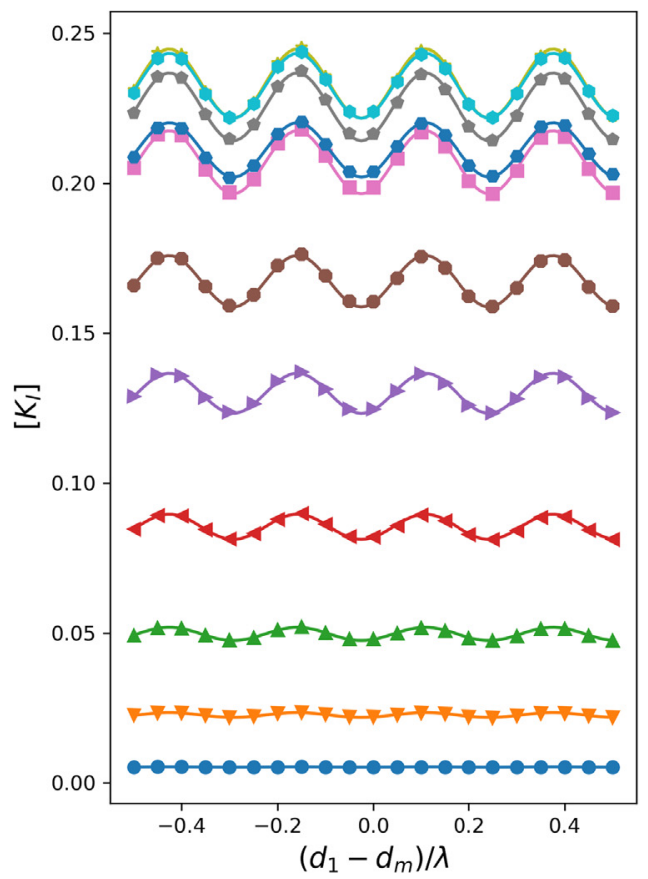

- $R_{c}=50.0 \mu \mathrm{m}$

$y=0.005+0.000 \sin (23.544 x-0.996)$

₹ $R_{c}=100.0 \mu \mathrm{m}$

$y=0.023+0.001 \sin (23.520 x-0.985)$

$R_{c}=150.0 \mu \mathrm{m}$

- $y=0.050+0.002 \sin (23.518 x-0.983)$

$R_{c}=200.0 \mu \mathrm{m}$

$y=0.085+0.004 \sin (23.517 x-0.982)$

$R_{c}=250.0 \mu \mathrm{m}$

- $y=0.130+0.007 \sin (23.515 x-0.982)$

$R_{c}=300.0 \mu \mathrm{m}$

$y=0.167+0.009 \sin (23.515 x-0.983)$

$R_{c}=400.0 \mu \mathrm{m}$

- $y=0.207+0.011 \sin (23.514 x-0.984)$

$R_{c}=500.0 \mu \mathrm{m}$

- $y=0.225+0.011 \sin (23.514 x-0.985)$

$R_{c}=600.0 \mu \mathrm{m}$

$R_{C}=600.0 \mu \mathrm{m}$
$y=0.233+0.012 \sin (23.513 x-0.986)$

- $R_{c}=1000.0 \mu \mathrm{m}$

- $y=0.233+0.011 \sin (23.510 x-0.991)$

- $R_{C}=1500.0 \mu \mathrm{m}$

- $y=0.211+0.009 \sin (23.508 x-0.998)$

Fig. 12. Dimensionless stress intensity factor $\left[K_{I}\right]$ as a function of $d_{1}$ in the neighborhood of $d_{m}=20 \mu m$.

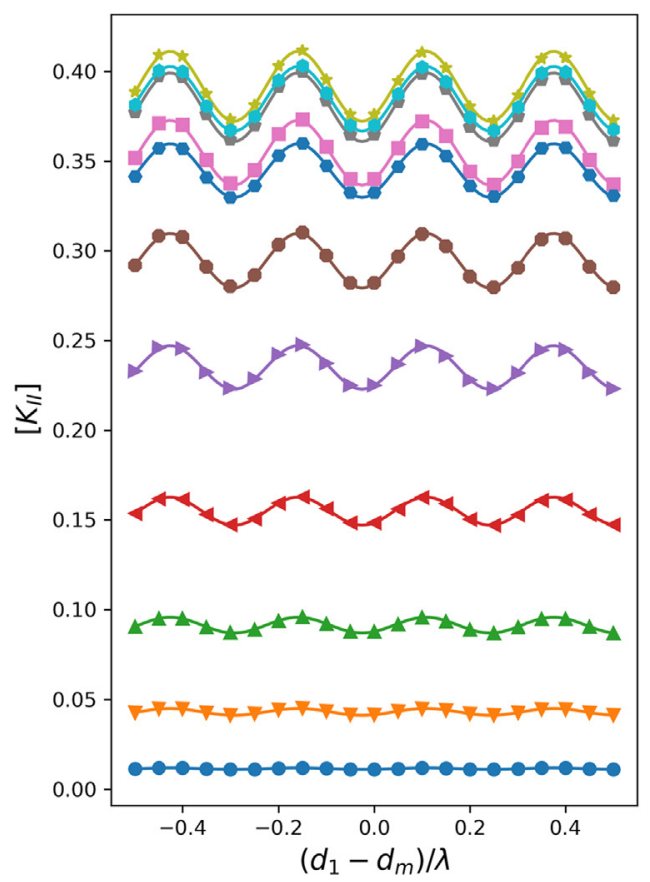

$R_{c}=50.0 \mu \mathrm{m}$
$y=0.011+0.000 \sin (23.512 x-0.986)$

$R_{c}=100.0 \mu \mathrm{m}$

$\nabla \begin{aligned} & R_{c}=100.01 m \\ & y=0.043+0.002 \sin (23.512 x-0.982)\end{aligned}$

$R_{c}=150.0 \mu m$

$\Delta=0.091+0.004 \sin (23.513 x-0.980)$

$R_{c}=200.0 \mu \mathrm{m}$

$y=0.155+0.008 \sin (23.513 x-0.979)$

$R_{c}=250.0 \mu \mathrm{m}$

$y=0.235+0.012 \sin (23.512 x-0.979)$

$R_{c}=300.0 \mu \mathrm{m}$

- $y=0.294+0.015 \sin (23.512 x-0.979)$

$R_{c}=400.0 \mu \mathrm{m}$

$y=0.355+0.018 \sin (23.513 x-0.978)$

$R_{c}=500.0 \mu \mathrm{m}$

- $y=0.380+0.019 \sin (23.513 x-0.979)$

R $R_{c}=600.0 \mu \mathrm{m}$

औ $y=0.392+0.019 \sin (23.512 x-0.979)$

$R_{c}=1000.0 \mu \mathrm{m}$

$y=0.385+0.018 \sin (23.510 x-0.983)$

$R_{c}=1500.0 \mu \mathrm{m}$

$y=0.345+0.015 \sin (23.507 x-0.989)$

Fig. 13. Dimensionless Stress intensity factor $\left[K_{I I}\right]$ as a function of $d_{1}$ in the neighborhood of $d_{m}=20 \mu m$.

Sinusoidal oscillation patterns similar to that of temperature peaks can be found again for both $K_{I}$ and $K_{I I}$ in the neighborhood of $d_{m}=20 \mu \mathrm{m}$, for every given crack radius. Therefore, the general sinusoidal function Eq. (27) can also be utilized for data fitting and the fitted expressions are obtained as shown in the right legends in Figs. 12 and 13. It is implied that the slight fluctuations of crack location would result in obvious change of stress intensity factors, which determine the stability of the defects embedded in the film. As we know, the crack would propagate further were the stress intensity factor greater than the fracture toughness of the material. Moreover, it is shown that the magnitudes of both $K_{I}$ and $K_{I I}$ are comparable to the fracture toughness of AZO, which indicates that the fracture would be of mixed mode. 


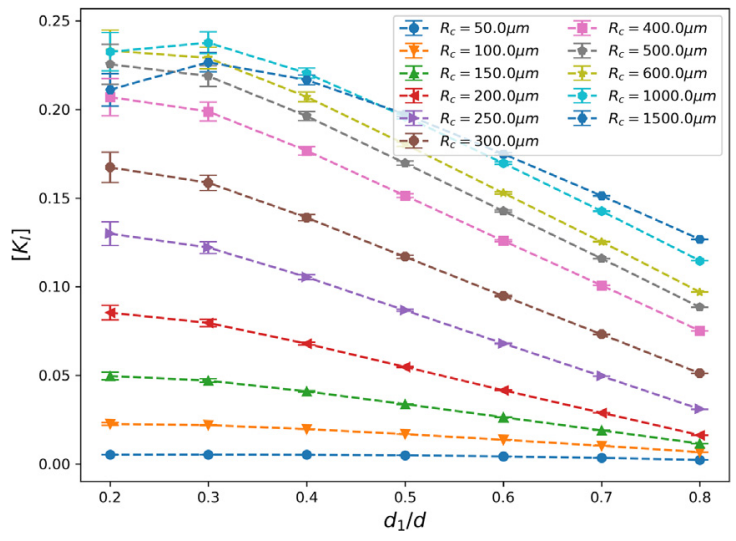

Fig. 14. Dimensionless Stress intensity factor $\left[K_{I}\right]$ as a function of $d_{1} / d$ in the overall range.

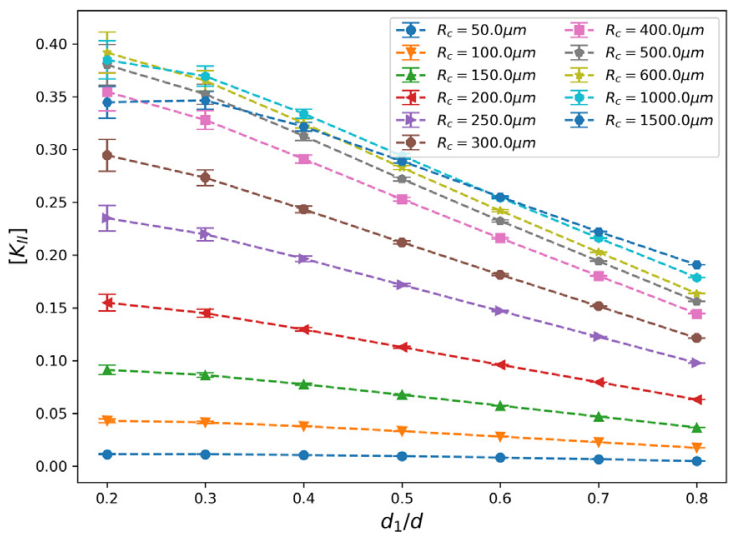

Fig. 15. Dimensionless Stress intensity factor $\left[K_{I I}\right]$ as a function of $d_{1} / d$ in the overall range.

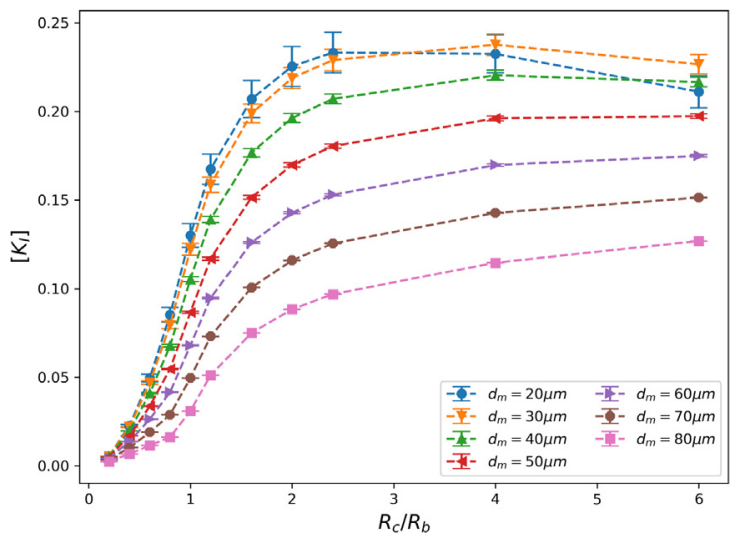

Fig. 16. Dimensionless Stress intensity factor $\left[K_{I}\right]$ as a function of $R_{c} / R_{b}$.

Figs. 14 and 15 show the fluctuations of $K_{I}$ and $K_{I I}$ as $d_{1}$ changes from $10 \mu \mathrm{m}$ to $90 \mu \mathrm{m}$, in which the mid-value and oscillation amplitudes are adopted considering the strong oscillation characteristic of both $K_{I}$ and $K_{I I}$ in the vicinity of any given crack location $d_{1}$.

The dashed lines show the relations of $K_{I}$ and $K_{I I}$ with $d_{1}$ for various crack radius and the error bars the oscillation amplitudes. It is shown that the oscillation amplitudes of both $K_{I}$ and $K_{I I}$ decay with increasing in $d_{1}$ for any given crack radius $R_{c}$. It is also shown that on the whole, the intensity factors decrease with increasing of crack depth $d_{1}$, although a small inflection point appears around the location $d_{1} / d=0.3$. This could be due to the fact that the temperature gradient has its maximum therein, although the mid-value of maximum temperature is monotonically decrease with increasing of $d_{1} / d$ as shown in Fig. 8.

Figs. 16 and 17 show the mid-value of the stress intensity factors $K_{I}$ and $K_{I I}$ as functions of the crack radius. Again, the error bars 


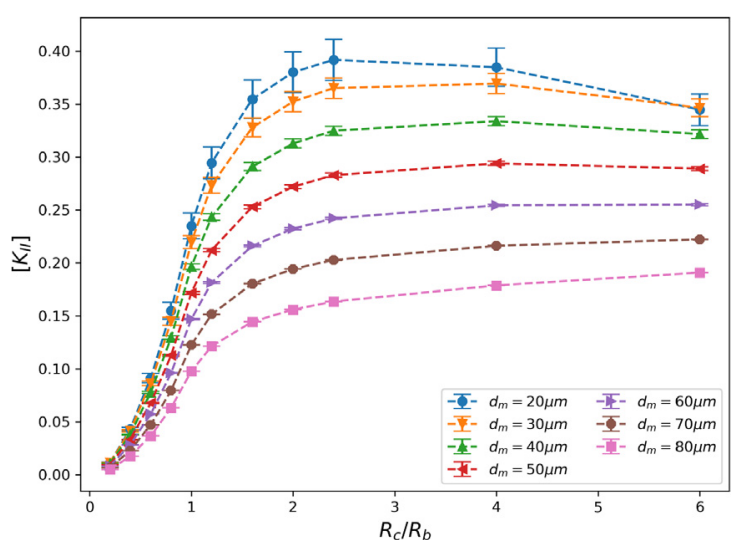

Fig. 17. Dimensionless Stress intensity factor $\left[K_{I I}\right]$ as a function of $R_{c} / R_{b}$.

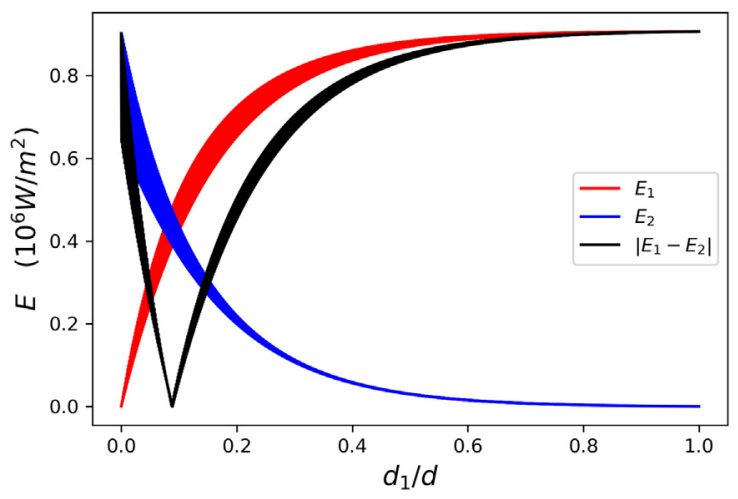

Fig. 18. Energy Fluxes versus $d_{1} / d$.

indicates the oscillation amplitude of the stress intensity factors. It is shown that the stress intensity factors increase with the increasing of crack radius $R_{c}$ in almost all of the present parameter range, which could be divided in two stages according to the climbing rates. The stress intensity factors increase quickly before $R_{c} / R_{b}=1$, then relatively slowly after $R_{c} / R_{b}>1$.

There is also a small deviation from the overall tendency when the crack locates near the irradiated surface, for which the midvalues of $K_{I}$ and $K_{I I}$ drop gradually after $R_{c} / R_{b}>2$ as indicated by the blue $d_{m}=20 \mu m$ line in Figs. 16 and 17 . Stress concentrations in these cases are determined by various amounts of energy absorption of the layers. The energy fluxes as a function of crack position are shown in Fig. 18. When the crack is near the surface $\left(\mathrm{d}_{1}<10 \mu \mathrm{m}\right)$, most of the inflow laser energy is absorbed by the layer under the crack. When the crack moves deeper, the dominant absorption domain is then $D_{1}$, the layer above the crack. Roughly speaking, since the thermal stress is induced by the temperature gradient, stress concentration can be related to both the local heat generation and the uneven absorption of inflow energy by the layers above and below the crack. When the crack tip is in the vicinity of the laser spot, the local heat flux is dominated by the local heat generation. On the contrary, when the crack tip is far away from the energy source, the uneven absorption becomes a more dominant factor than the local heat generation to the stress concentration around the crack tip. In that case, the stress intensity factor is to a large extent determined by the absolute difference of absorption, $\left|E_{1}-E_{2}\right|$, which decreases with an increasing $d_{1} / d$ to the minimal value around $d_{1} / d \approx 0.1$, and then increases rapidly to a limiting value in $d_{1} / d \in[0.2,0.4]$. Such effect accounts for the different tendency of $K_{I}$ and $K_{I I}$ to $d_{1} / d$ at small $d_{1} / d$ and large $R_{c} / R_{b}$ than others.

\section{Conclusions}

A subsurface crack in the transparent conducting film would induce multiple reflection, transmission, interference, absorption and therefore complex heat dissipation from absorbed light energy. The volumetric heat generation rate in the upper part of the film above the crack would harmonically fluctuate, in particular when the crack location is close to the irradiated surface. That is, the fluctuation amplitude of the volumetric heat generation rate would quickly decrease were the crack location farther away from upper surface. Such fluctuation pattern in heat generation results in similar fluctuations of the temperature peaks and stress intensity factors. Averagely speaking, the peak temperatures and stress intensity decrease gradually with the increasing in the distances of the crack location from the upper surface. However, the temperature peaks and stress intensity factor increase with increasing in the crack lengths, both of which implies some ultimate steady magnitudes if the crack length is long enough in comparison with laser beam radius. 


\section{Acknowledgements}

This work was supported by the National Natural Science Foundation of China (Grant Nos. 11572327 and 11332011 ) and the Strategic Priority Research Program of Chinese Academy of Sciences (Grant No. XDA17030200).

\section{References}

[1] Abrinaei F, Shirazi M, Hosseinnejad MT. Investigation of growth dynamics of nanostructured aluminum doped zinc oxide thin films deposited for the solar cell applications. J Inorg Organomet Polym Mater 2016;26:233-41.

[2] Belghachem N, Mlynczak J, Kopczynski K, Mierczyk Z, Gawron M. Thermal analysis of a diffusion bonded Er3 +, Yb3 + :glass/Co2 + : MgAl2O4 microchip lasers. Opt Mater 2016;60:546-51.

[3] Bouderbala M, Hamzaoui S, Stambouli AB, Bouziane H. Effects of the position substrate upon the structural behaviour, electrical and optical properties of zincoxide films used in solar cells. Appl Energy 1999;64:89-96.

[4] Cai YC, Wang ML, Zhang HZ, Yang LJ, Fu XH, Wang Y. Laser cutting sandwich structure glass-silicon-glass wafer with laser induced thermal-crack propagation. Opt Laser Technol 2017;93:49-59.

[5] Chai H. Crack propagation in glass coatings under expanding spherical contact. J Mech Phys Solids 2006;54:447-66.

[6] Chen B, Meng C, Yang Z, Li W, Lin S, Gu T, et al. Graphene coated ZnO nanowire optical waveguides. Opt Express 2014;22:24276-85.

[7] Chiu J-M, Chu C-C, Zena DM, Tai Y. Simultaneous enhancement of photocurrent and open circuit voltage in a ZnO based organic solar cell by mixed selfassembled monolayers. Appl Energy 2015;160:681-6.

[8] El-Brolossy TA, Saber O, Ibrahim SS. Determining the thermophysical properties of Al-doped ZnO nanoparticles by the photoacoustic technique. Chin Phys B 2013;22:074401.

[9] Hosseinabadi S, Abrinaei F, Shirazi M. Statistical and fractal features of nanocrystalline AZO thin films. Phys A 2017;481:11-22.

[10] Huang B, Zhao M-H, Zhang T-Y. Indentation fracture and indentation delamination in ZnO film/Si substrate systems. Phil Mag 2004;84:1233-56.

[11] Huang Y-C, Chang S-Y. Substrate effect on mechanical characterizations of aluminum-doped zinc oxide transparent conducting films. Surf Coat Technol 2010;204:3147-53.

[12] Jamil MSC, Fauzi ERI, Juinn CS, Sheikh MA. Laser bending of pre-stressed thin-walled nickel micro-tubes. Opt Laser Technol 2015;73:105-17.

[13] Johnson JC, Yan H, Yang P, Saykally RJ. Optical cavity effects in ZnO nanowire lasers and waveguides. J Phys Chem B 2003;107:8816-28.

[15] Klinsmann M, Rosato D, Kamlah M, McMeeking RM. Modeling crack growth during Li insertion in storage particles using a fracture phase field approach. J Mech Phys Solids 2016;92:313-44.

[16] Khosrownejad SM, Curtin WA. Crack growth and fracture toughness of amorphous Li-Si anodes: mechanisms and role of charging/discharging studied by atomistic simulations. J Mech Phys Solids 2017;107:542-59.

[17] Kumarakuru H, Cherns D, Collins AM. The growth and conductivity of nanostructured ZnO films grown on Al-doped ZnO precursor layers by pulsed laser deposition. Ceram Int 2014;40:8389-95.

[18] Kumar A, Huang N, Staedler T, Sun C, Jiang X. Mechanical characterization of aluminum doped zinc oxide (Al:ZnO) nanorods prepared by sol-gel method. Appl Surf Sci 2013;265:758-63.

[19] Kumar S. Selective laser sintering: a qualitative and objective approach. J Miner, Metals Mater Society (TMS) 2003;55:43-7.

[20] Li HN, Yu TB, Zhu LD, Wang WS. Evaluation of grinding-induced subsurface damage in optical glass BK7. J Mater Process Technol 2016;229:785-94.

[21] Lin L-Y, Jeong M-C, Kim D-E, Myoung J-M. Micro/nanomechanical properties of aluminum-doped zinc oxide films prepared by radio frequency magnetron sputtering. Surf Coat Technol 2006;201:2547-52.

[22] Lindqvist M, Louter C. Experimental study on glass edge machining flaw characterization. Engng Fract Mech 2014;127:56-70.

[23] Maldonado F, Stashans A. Al-doped ZnO: electronic, electrical and structural properties. J Phys Chem Solids 2010;71(5):784-7.

[24] Manenkov AA. Fundamental mechanisms of laser-induced damage in optical materials: today's state of understanding and problems. Opt Eng 2014;53(1):010901.

[25] Ozgowicz W, Grzegorczyk B, Pawełek A, Wajda W, Skuza W, Piątkowski A, et al. An analysis of the Portevin-Le Chatelier effect and cracking of CuSn6P alloy at elevated temperature of deformation applying the acoustic emission method. Eng Fract Mech 2016;167:112-22.

[26] Peng Q. An analytical solution for a transient temperature field during laser heating a finite slab. Appl Math Modell 2016;40:4129-35.

[27] Sakai K, Nonaka K. Note: detection of micro-cracks in the interlayer dielectric film by the stress based light scattering method. Rev Sci Instrum 2011;82:116103.

[28] Sakata Y, Terasaki N, Sakai K, Nonaka K. Origin of light scattering variations of a latent flaw through light scattering measurement with applied stress effect. Jpn J Appl Phys 2016;55:118002.

[29] Shuja SZ, Yilbas BS. Laser heating of a moving slab: Influence pulse intensity parameter on temperature and stress fields. Opt Laser Technol 2015;70:7-16.

[31] Taabouche A, Bouabellou A, Kermiche F, Hanini F, Sedrati C, Bouachiba Y, et al. Preparation and characterization of Al-doped ZnO piezoelectric thin films grown by pulsed laser deposition. Ceram Int 2016;42:6701-6.

[32] Vedrtnam A, Pawar SJ. Laminated plate theories and fracture of laminated glass plate - A review. Eng Fract Mech 2017;186:316-30.

[33] Voss T, Svacha GT, Mazur E. High-order waveguide modes in ZnO nanowires. Nano Lett 2007;7:3675-80.

[34] Wang J, Li YG, Han JH, Xu Q, Guo YB. Evaluating subsurface damage in optical glasses. J Eur Opt Soc - Rapid Publ 2011;6:11001.

[35] Wang Q, Wang Y, Chen H, Xiao H, Sun J, He L. Frame constraint effect on the window glass crack behavior exposed to a fire. Eng Fract Mech 2013;108:109-19.

[36] Wu C-W, Peng Q, Huang C-G. Thermal analysis on multijunction photovoltaic cell under oblique incident laser irradiation. Energy 2017;134:248-55.

[37] Yahata K, Yamamoto K, Ohmura E. Crack propagation analysis in laser scribing of Glass. JLMN-J Laser Micro/Nanoeng 2010;5(2):109-14.

[38] Yuan Y-C, Wu C-W. Thermal analysis of film photovoltaic cell subjected to dual laser beam irradiation. Appl Therm Eng 2015;88:410-7.

[39] Zhao B, Zikry MA. Oxidation-induced failure in semi-crystalline organic thin films. Int J Solids Struct 2017;109:72-83.

[40] Zhang L, Chen W, Hu LL. Systematic investigation on light intensification by typical subsurface cracks on optical glass surfaces. Appl Opt 2013;52(5):980-9. 